\title{
A Plant-wide Case for Control System Study in Teaching of Process Control Engineering
}

\author{
Lingjian $Y e^{1, a}$ and Hongwei Guan ${ }^{2, b^{*}}$ \\ ${ }^{1}$ Ningbo Institute of Technology, Zhejiang University, Ningbo 315100, Zhejiang, China \\ ${ }^{2}$ Ningbo Dahongying University, 315175, Ningbo, Zhejiang, China \\ aljye@nit.net.cn, b ghw_nit@126.com
}

Keywords: Control system design; Process control engineering; Practical teaching; Simulation

\begin{abstract}
In the process control engineering course, control methods are often introduced separately, which lacks an efficient way to deliver knowledge of control integration through case studies. In this paper, we introduce a plant-wide benchmark platform, the Tennessee Eastman (TE) process, for the teaching of process control engineering. In the control system of TE process, there are substantial control configurations with different control methods/strategies, including the classic PID control, ratio control, cascade control, override control, feedforward control, and so on. Using a uniform case study, it is easier to build a conception how different control strategies are coordinated to operate a realistic plant.
\end{abstract}

\section{Introduction}

The process control engineering course is an important course for students majoring in Automation, which is concerned with control methods and strategies that are widely used in industrial applications. A wide range of control methods are included in the course, for example, the classic PID control, ratio control, cascade control, override control, feedforward control, and so on. However, these subjects are often introduced separately under different applications, such as the numerical examples and small-scale plants. Over years of teaching expericences, we found that it is inefficient to deliver the scattered knowledge points. Because for each method, the students need to get familiar with a new practice, and finally they lose the big picture how they are integrated for controlling a true plant-wide process.

In literature, the Tennessee Eastman (TE) process [1] is found to be an excellent case to serve our teaching purpose. The TE process is a simulated large-scale chemical plants and has been widely investigated as a benchmark for both acadamic and educational purposes [2-7]. Many researchers have proposed control systems as solutions to operate the plant [2-7], among which the one designed by Ricker [6] is very efficient and prevalent. The control system is a decentralized one, which was designed with both quantitive analysis and expert knowledge. Overall, the whole control system contain substantial control methods/trategies, including all the ones that the course is of interest. Therefore, the course can be taken around this large-scale simulation platform, which is easier to concentrate hence improving the education efficiency.

\section{Introductions of the TE Process}

Overview. The simulated TE process is developed based on a real chemical plant [1]. It has 5 major operating units: the reactor, a product condenser, a vapor-liquid separator, a recycle compressor and the product stripper. The flowsheet is shown in Fig.1. The process includes 12 manipulated variables (MVs) and 41 measurements, as listed in Table 1 and Table 2. Furthermore, the process is defined with six operating modes, which differ from the desired mixup of product $(\mathrm{G} / \mathrm{H}$ mass ratio) or whether the production rate is fixed or aimed to be maximized. Among the six operating modes, the first one (Mode 1) was considered most intensively. 


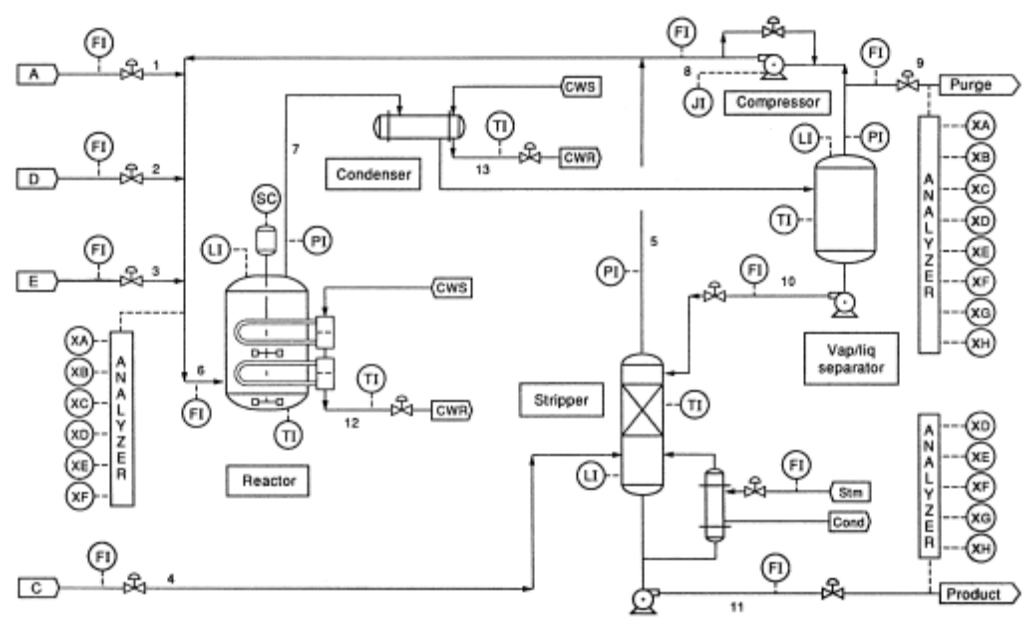

Figure 1. The TE process

Table 1 Manipulated variables of the TE process

\begin{tabular}{|l|l|}
\hline Number & Variable name \\
\hline XMV(1) & D feed flow \\
\hline XMV(2) & E feed flow \\
\hline XMV(3) & A feed flow \\
\hline XMV(4) & A and C feed flow \\
\hline XMV(5) & Compressor recycle valve \\
\hline XMV(6) & Purge valve \\
\hline XMV(7) & Separator liquid flow \\
\hline XMV(8) & Stripper liquid product flow \\
\hline XMV(9) & Stripper steam valve \\
\hline XMV(10) & Reactor cooling water flow \\
\hline XMV(11) & Condenser cooling water flow \\
\hline XMV(12) & Agitator speed \\
\hline
\end{tabular}

Control System. In the control system designed by Ricker [6], the following process variables are controlled: Separator level, stripper level, production rate, product quality (mole \%G in product), reactor pressure (maximum) and level (minimum), compressor recycle valve (closed), stripper steam valve (closed), agitator speed (maximum), reactor temperature, $\% \mathrm{~A}$ and $\% \mathrm{C}$ in the feed. These controlled variables are assigned with appropriate MVs and controlled in a decentralized control structure.

Table 2 Measurements of the TE process

\begin{tabular}{|c|c|c|c|}
\hline Number & Variable name & Number & Variable name \\
\hline XMEAS(1) & A feed & XMEAS(14) & Product separator underflow \\
\hline XMEAS(2) & D feed & XMEAS(15) & Stripper level \\
\hline XMEAS(3) & E feed & XMEAS(16) & Stripper pressure \\
\hline XMEAS(4) & $\mathrm{A}$ and $\mathrm{C}$ feed & XMEAS(17) & Stripper underflow \\
\hline XMEAS(5) & Recycle flow & XMEAS(18) & Stripper temperature \\
\hline XMEAS(6) & Reactor feed rate & XMEAS(19) & Stripper steam flow \\
\hline XMEAS(7) & Reactor pressure & XMEAS(20) & Compressor work \\
\hline XMEAS(8) & Reactor level & XMEAS(21) & Reactor cooling water outlet temperature \\
\hline XMEAS(9) & Reactor temperature & XMEAS(22) & Separator cooling water temperature \\
\hline XMEAS(10) & Purge rate & XMEAS(23-28) & mole fraction of $\mathrm{A}-\mathrm{F}$ in feed \\
\hline XMEAS(11) & Separator temperature & XMEAS(29-36) & mole fraction of $\mathrm{A}-\mathrm{H}$ in purge \\
\hline XMEAS(12) & Separator level & XMEAS(37-41) & mole fraction of $\mathrm{D}-\mathrm{H}$ in product \\
\hline XMEAS(13) & Separator pressure & & \\
\hline
\end{tabular}




\section{Teaching based on the TE Process}

Various control methods and strategies are embeded in the control system of the TE process, which are exactly the main content of the process control engineering course. Therefore, the whole course can be conducted around the TE processes in a systematic way.

Classic PID Control. The classic PID control is the most widely used control algorithm in the TE control sytem, which is also the case in general industrial plants. The control of reactor temperature is of this type (PI), which is configured with the reactor coolant valve, XMV(10). The controller gain and integral time are set as -8.0 and 7.5 min, respectively.

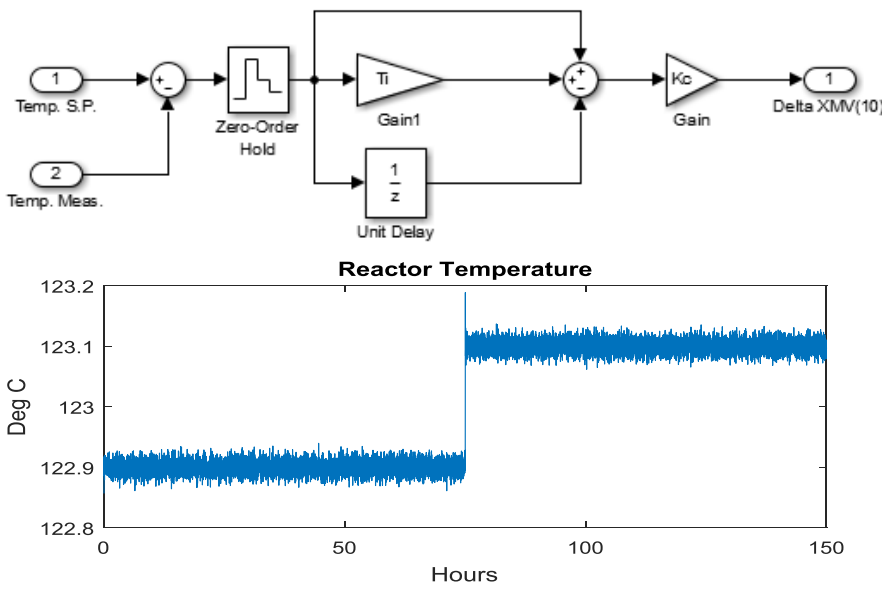

Figure 2. PI control of the reactor temperature

The control block in Simulink and simulation are shown in Fig.2, where at $75 \mathrm{~h}$ the setpoint is changed from $122.9{ }^{\circ} \mathrm{C}$ to $131.1^{\circ} \mathrm{C}$ to show the tracking performance. Note that the respose is very quick due to the well-tuned controllers.

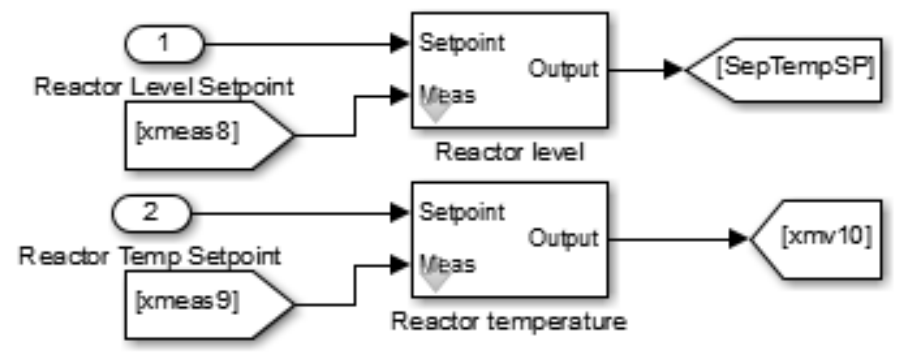

Figure 3. Cascade control of the reactor level

Cascade Control. The reactor level is fundamental for inventory control. In the control system, the reactor level is configured with cascade control, where the main control loop is the level controlled by separator temperature, and the slave loop is separator temperature controlled by condenser cooling water flow. The gains and integral time of the two controllers are $0.8,60 \mathrm{~min}$ and $-4.0,15 \mathrm{~min}$, respectively. The cascade structure is shown in Fig.3.

Another example is the treactor pressure, which is one of the most important control objective in the TE process. If the reactor pressure exceeds $3000 \mathrm{~Pa}$, the process shuts down due to safety consideration. The control loop is configured with cascade control, where the main control loop is pressure controlled by $r_{5}$ (intermediate variable), and the slave control loop is the purge rate controlled by associated valve, $\mathrm{XMV}(6)$. The tracking performance of reactor pressure is shown in Fig.4, where at $80 \mathrm{~h}$ the setpoint is ramped from $2800 \mathrm{kPa}$ to $2650 \mathrm{kPa}$. 


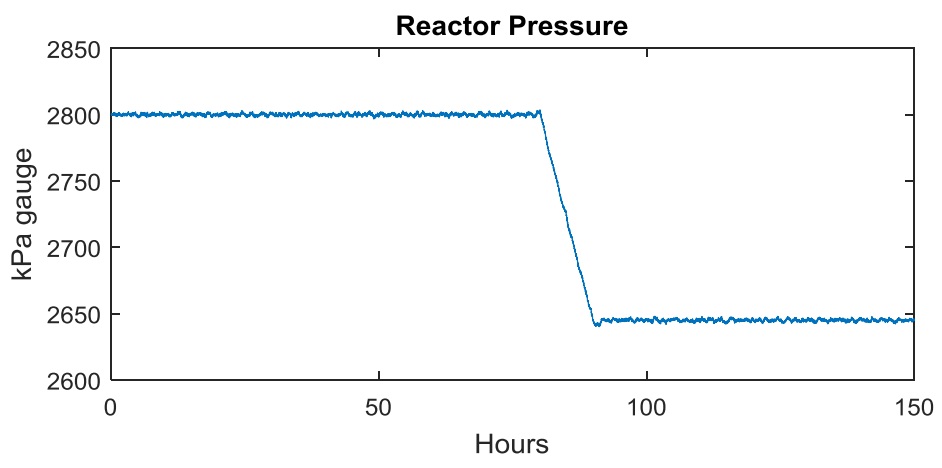

Figure 4. Tracking the reactor pressure

Feedforward Control. The control system uses a number of feedforward calculations combined with feedback control to enhance the performance. As an example, in the control of production quality $(\% \mathrm{G})$, a lookup table is bulit determining parameters, $r_{2 F F}$ and $r_{3 F F}$, depending on the setpoints of $\% \mathrm{G}$. The combined feedforward-feedback strategy is:

$$
\begin{aligned}
& r_{2}(t)=r_{2 F F}(t)-\frac{32 E_{a d j}(t)}{F_{p}(t)} \\
& r_{3}(t)=r_{3 F F}(t)-\frac{46 E_{a d j}(t)}{F_{p}(t)}
\end{aligned}
$$

where $E_{\text {adj }}$ is an intermediate signal representing the adjustment to the molar feed rate of $\mathrm{E}, F_{\mathrm{p}}$ is $\mathrm{s}$ a production index, whose value is 100 corresponding to $23.0 \mathrm{~m}^{3} \cdot \mathrm{h}^{-1}$ (base case). $E_{\text {adj }}$ is configured in a feedback loop to control \% G, and then $\mathrm{Eq}(1)$ and $\mathrm{Eq}$ (2) are used combined with the aforementioned lookup table to calcute $r_{2}$ and $r_{3}$, which are further configured in two feedback loops by manipulating the feedrates of D and E. Fig. 5 shows the tracking performance of $\% \mathrm{G}$, where at $75 \mathrm{~h}$ the setpoint is changed from $53.8 \%$ to $54.8 \%$.

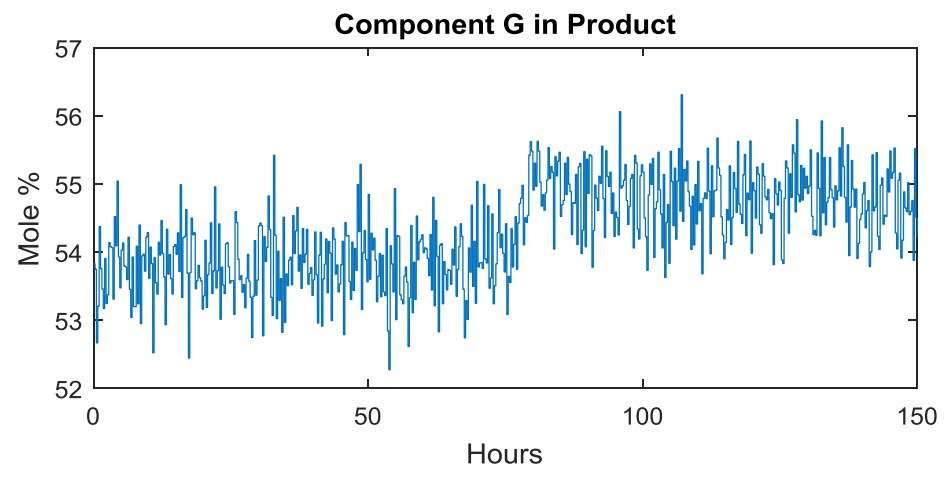

Figure 5. Tracking the production quality $(\% \mathrm{G})$

Override Control. The overide control is to handle special cases that can not be addressed with regular control system. Particular control philosophy is used, often a logical strategy. In the TE system, there are several cases with override control, for example:

(1) When the loop 12 controlling the reactor pressure saturates, the production rate target, $F_{p}$ is reduced. In this way, the override loop keeps the pressure less than the shutdown limit of $3000 \mathrm{kPa}$ under all conditions;

(2) In the control loop of reactor liquid level with the separator temperature, the condenser coolant valve may saturate. One override solution is, if a high reactor liquid level persists, the setpoint of separator temperature decreases, and the condenser valve is $100 \%$ open.

Ratio Control. \% A and \% $\mathrm{C}$ in the feed are controlled in the TE system. However, they are not directly used as the controlled variables, rather, the quantities $y_{A}=\% \mathrm{~A}+\% \mathrm{C}$ and $y_{A C}=\% \mathrm{~A} /(\% \mathrm{~A}+\% \mathrm{C})$ in the feed are controlled. This is due to the fact that stream 1 is pure A, so it mainly influences the relative 
amount of $\mathrm{A}$ The sum of streams 1 and 4 determines the amount of $\mathrm{A}+\mathrm{C}$ relative to the other components.

Teaching focus. Above subjects constitute the main knowledge points for the course of process control enginnering. During the teaching, the effect of parameter tuning, disturbance rejection and setpoint tracking can be illustrated through simulations. Comparisons among different methods are helpful to demonstrate their pros and cons, which can be easily carried out in the TE platform.

\section{Summary}

In this study, we recommended using the simulation benchmark, namely the TE process, for teaching the course of process control enginnering. The plant-wide TE process was configured with substantial control methods/strategies, which cross over the whole course. Using a uniform case study, it is easier to build the conception how different control strategies are coordinated to better operate a real plant.

\section{Acknowledgements}

This work is supported by Ningbo Education Planning Project (2016YGH032) and Ningbo Natural Science Foundation (2015A610151).

\section{References}

[1] J. J. Downs, E. F. Vogel, A plant-wide industrial process control problem, Comput. Chem. Eng., Vol. 17(1993), No. 3, pp. 245-255.

[2] T. McAvoy, N. Ye, Base control for the tennessee eastman problem, Computers \& Chemical Engineering, Vol. 18(1994), No. 5, pp. 383-413.

[3] N. Ricker, Optimal steady-state operation of the Tennessee-Eastman challenge process, Computers \& Chemical Engineering, Vol. 19(1995), No. 9, pp. 949-959.

[4] N. Ricker, J. Lee, Nonlinear model predictive control of the Tennessee Eastman challenge process, Computers \& Chemical Engineering, Vol. 19(1995), No. 9, pp. 961-981.

[5] N. Ricker and J. Lee, Nonlinear modeling and state estimation for the Tennessee Eastman challenge process, Computers \& chemical engineering, Vol. 19(1995), No. 9, pp. 983-1005.

[6] N. Ricker, Decentralized control of the Tennessee Eastman challenge process, J. Process Control, Vol. 6(1996), No. 4, pp. 205-221.

[7] A. Jha, O. C. Okorafor, Optimal plantwide process control applied to the Tennessee Eastman problem, Industrial \& Engineering Chemistry Research, Vol. 53(2013), No. 2, pp. 738-751. 